\title{
Efficacy of a Probiotic Consisting of Lacticaseibacillus rhamnosus PDV 1705, Bifidobacterium bifidum PDV 0903, Bifidobacterium longum subsp. infantis PDV 1911, and Bifidobacterium longum subsp. longum PDV 2301 in the Treatment of Hospitalized Patients with COVID-19: a Randomized Controlled Trial
}

\author{
Vladimir Ivashkin ${ }^{1,2} \cdot$ Victor Fomin $^{1} \cdot$ Sergey Moiseev $^{1} \cdot$ Michail Brovko $^{1} \cdot$ Roman Maslennikov $^{1,2} \mathbb{D}$. \\ Anatoly Ulyanin $^{1,2}$. Victoria Sholomova ${ }^{1}$ - Maria Vasilyeva ${ }^{1}$. Elizaveta Trush ${ }^{1}$. Oleg Shifrin ${ }^{1}$. Elena Poluektova ${ }^{1,2}$
}

Accepted: 5 October 2021

() The Author(s), under exclusive licence to Springer Science+Business Media, LLC, part of Springer Nature 2021

\begin{abstract}
The treatment of coronavirus disease (COVID-19) and COVID-19-associated diarrhea remains challenging. This study aimed to evaluate the efficacy of a multi-strain probiotic in the treatment of COVID-19. This was a randomized, controlled, singlecenter, open-label trial (NCT04854941). Inpatients with confirmed COVID-19 and pneumonia were randomly assigned to a group that received a multi-strain probiotic (PRO group) or to the control group (CON group). There were 99 and 101 patients in the PRO and CON groups, respectively. No significant differences in mortality, total duration of disease and hospital stay, incidence of intensive care unit admission, need for mechanical ventilation or oxygen support, liver injury development, and changes in inflammatory biomarker levels were observed between the PRO and CON groups among all included patients as well as among subgroups delineated based on age younger or older than 65 years, and subgroups with chronic cardiovascular diseases and diabetes. Diarrhea on admission was observed in $11.5 \%$ of patients; it resolved earlier in the PRO group than in the CON group (2 [1-4] vs. 4 [3-6] days; $p=0.049$ ). Hospital-acquired diarrhea developed less frequently in the PRO group than in the CON group among patients who received a single antibiotic ( $0 \%$ vs. $12.5 \% ; p=0.023)$ unlike among those who received $>1$ antibiotic $(10.5 \%$ vs. $13.3 \% ; p=0.696)$. The studied probiotic had no significant effect on mortality and changes in most biomarkers in COVID-19. However, it was effective in treating diarrhea associated with COVID-19 and in preventing hospital-acquired diarrhea in patients who received a single antibiotic.
\end{abstract}

Keywords COVID-19 $\cdot$ Probiotics $\cdot$ Diarrhea $\cdot$ Mortality $\cdot$ Liver

\section{Introduction}

Coronavirus disease 2019 (COVID-19) is an acute respiratory infection with systemic manifestations [1]. Despite intensive research, COVID-19 treatment remains an important challenge [2]. Probiotics are live microorganisms that, when administered in adequate amounts, confer health

Roman Maslennikov

mmmm00@yandex.ru

1 Department of Internal Medicine, Gastroenterology and Hepatology, Sechenov University, Moscow, Russian Federation

2 Scientific Community for Human Microbiome Research, Moscow, Russian Federation benefits on the host [3]. They have been reported to show a positive effect on acute respiratory infections by modulating immune responses [4].

Diarrhea is a manifestation of COVID-19, and has been reported in approximately 10\% of COVID-19 patients [5]. COVID-19-associated diarrhea has distinct characteristics [6], and may even be the first manifestation of COVID-19 [7]. Although the exact mechanisms of the development of diarrhea in COVID-19 remain unknown, the following factors of its pathogenesis can be suggested. The viral E protein binds to proteins of the tight junctions of enterocytes, which leads to an increase in the permeability of the intestinal barrier, bacterial translocation, and gut inflammation. Moreover, the viral proteins E and Orf3a also disrupt the functioning of ion channels in the enterocyte. In addition, 
the pathogenesis of diarrhea in COVID-19 patients may also include intestinal damage during a cytokine storm, gut dysbiosis, and Clostridioides difficile superinfection [8]. A number of probiotics have been shown to be effective in the treatment of diarrhea of various etiologies [9-11].

Liver injury develops in an average of 20-25\% of COVID19 patients [12]. Several probiotics have been shown to be effective in the treatment of certain liver diseases [13].

Many experts have suggested the use of probiotics in combination with other drugs for the management of COVID-19 [14-21].

The effect of multi-strain probiotics on COVID-19 has been evaluated in three published retrospective studies [22-24]. Li et al. reported that the administration of a probiotic including Bifidobacterium longum subsp. infantis, Bifidobacterium longum subsp. longum, Lactobacillus acidophilus, Lactobacillus delbrueckii subsp. bulgaricus, Bacillus cereus, Bacillus subtilis, Streptococcus thermophiles, and Enterococcus faecium led to a slight decrease in hospitalization duration and accelerated the clearance of severe acute respiratory syndrome coronavirus 2 (SARSCoV-2), the causative agent of COVID-19 [22]. Ceccarelli et al. showed that the use of a probiotic including $S$. thermophilus DSM 32,245, Bifidobacterium lactis DSM 32,246, Bifidobacterium lactis DSM 32,247, L. acidophilus DSM 32,241, L. helveticus DSM 32,242, Lacticaseibacillus paracasei DSM 32,243, L. plantarum DSM 32,244, and L. brevis DSM 27,961 reduced mortality in COVID-19 patients [23]. D'Ettorre et al. described the positive effects of the same probiotic on diarrhea associated with COVID-19 [24].

However, these findings have not been verified in prospective randomized studies. The aim of our study was to evaluate the effect of a multi-strain probiotic on mortality, disease course, respiratory function, diarrhea, and liver injury in COVID-19 patients in a randomized controlled trial.

\section{Materials and Methods}

This was a randomized, controlled, single-center, openlabel trial. All patients signed an informed consent for study participation and the use of off-label drugs. The study was approved by the local ethics committee (Conclusion №. 34-20 of September 9, 2020) in accordance with the Declaration of Helsinki, and was registered at https://clinicaltrials. gov (NCT04854941). The research protocol can be accessed at this website as well. The study received no funding.

\section{Patients}

The study included patients with COVID-19 admitted to the Clinic of Internal Diseases of Sechenov University. COVID-19 was confirmed using polymerase chain reaction on nasopharyngeal and oropharyngeal swabs to detect SARSCoV-2. The study was conducted from December 2020 to March 2021 and included participants in the age range from 18 to 75 years.

The exclusion criteria were as follows: age over 75 years or under 18 years, consumption of probiotics for 3 months prior to admission, presence or history of intolerance to probiotics or their components, refusal to participate and sign informed consent, pregnant or breastfeeding, cancer or mental illness, and severe renal (glomerular filtration rate less than $50 \mathrm{~mL} /$ $\min$ ) or hepatic (equivalent to cirrhosis class $\mathrm{B}$ or $\mathrm{C}$ on the Child-Pugh scale) dysfunction at the time of admission.

Patients who prematurely discontinued the consumption of probiotics for reasons not related to the development of side effects were excluded from the study.

\section{Intervention}

The patients were randomized to the probiotics group (PRO group) or the control group (CON group). During the hospital stay, patients in the $\mathrm{PRO}$ group received the following probiotics three times a day for no more than 14 days: Florasan-D containing $\sim 10^{9}$ colony forming units (CFU) of Lacticaseibacillus rhamnosus PDV 1705, 109 CFU of Bifidobacterium bifidum PDV 0903, 10 $0^{9} \mathrm{CFU}$ of Bifidobacterium longum subsp. infantis PDV 1911, and $\sim 10^{9}$ CFU of Bifidobacterium longum subsp. longum PDV 2301. The end point of the trial was day 14 of hospitalization or the day of the patient's discharge or death, whichever occurred earlier. The follow-up period lasted from the time of the inclusion until recovery or death.

\section{Controls}

The control group consisted of patients who did not receive probiotics.

Patients in both the groups also received dexamethasone and antiviral (favipiravir and/or riamilovir), antibacterial, anticoagulant (enoxaparin in most cases; rivaroxaban and dabigatran were used less frequently), and anticytokine (tocilizumab or/and olokizumab) drugs according to indications and contraindications (Table S1).

\section{Outcomes}

Death from any cause, duration of hospitalization, total duration of the disease, incidence of admission to intensive care unit, need for oxygen support or mechanical ventilation, and changes in the values of key biomarkers were considered as the main outcomes. The duration of diarrhea [an increase in the frequency of bowel movements (more than three times per day)], incidence of hospital-acquired diarrhea, progression of pre-existing liver injury, and onset of liver injury were considered as additional outcomes. Liver injury was determined 
based on the presence of abnormalities in any of the main liver test findings (serum alanine transaminase, aspartate transaminase, alkaline phosphatase, gamma-glutamyl transferase, total bilirubin, and albumin levels).

The volume of the affected lungs was measured using chest computed tomography (CT); it included the sum of ground glass and consolidation volumes.

We performed per-protocol analysis as there was no final point to perform intention-to-treat analysis.

\section{Statistics}

Results are presented as median [interquartile range]. The groups were compared using the Mann-Whitney test for continuous data and chi-square test for categorical data. Wilcoxon test was used to assess changes in continuous biomarker values. Mortality was assessed using the Kaplan-Meier estimator and Cox's test. A $p$ value $\leq 0.05$ was considered as the criterion for significance. Statistical calculations were performed using STATISTICA 10 (TIBCO Software, Palo Alto, CA).

\section{Results}

\section{Characteristics of the Included Patients by Groups}

The study included 99 patients in the PRO group and 101 patients in the CON group (Fig. 1). COVID-19 pneumonia was confirmed in all patients using a chest CT scan. There was no significant difference in age, sex distribution, body mass index, body temperature at admission, symptoms of COVID-19, incidence of co-morbidities, and use of other drugs for the treatment of COVID-19 between the groups (Table 1; Table S1).

\section{Main Outcomes}

No significant differences in the total duration of disease, length of hospital stay, incidence of intensive care unit admission, need for mechanical ventilation or oxygen support (Table 1), and the changes in the volume of the affected lungs and serum levels of biomarkers of systemic inflammation [C-reactive protein (CRP), erythrocyte sedimentation rate, ferritin, fibrinogen, white blood cells, neutrophils, and lymphocytes], renal function (creatinine), and liver function (alanine aminotransferase, aspartate aminotransferase, albumin, and total bilirubin) were observed between the groups (Tables 2 and 3 ).

The patients in the PRO and CON groups had similar survival rates $(p=0.491)$ (Table 1 ; Fig. 2 a). Two patients who stopped taking the probiotic prematurely also survived.

As the presence of cardiovascular diseases or diabetes is a risk factor for poor prognosis in COVID-19 [25], an analysis was performed for these subgroups of patients. The administration of the probiotics had no significant effects
Fig. 1 CONSORT 2010 flow diagram

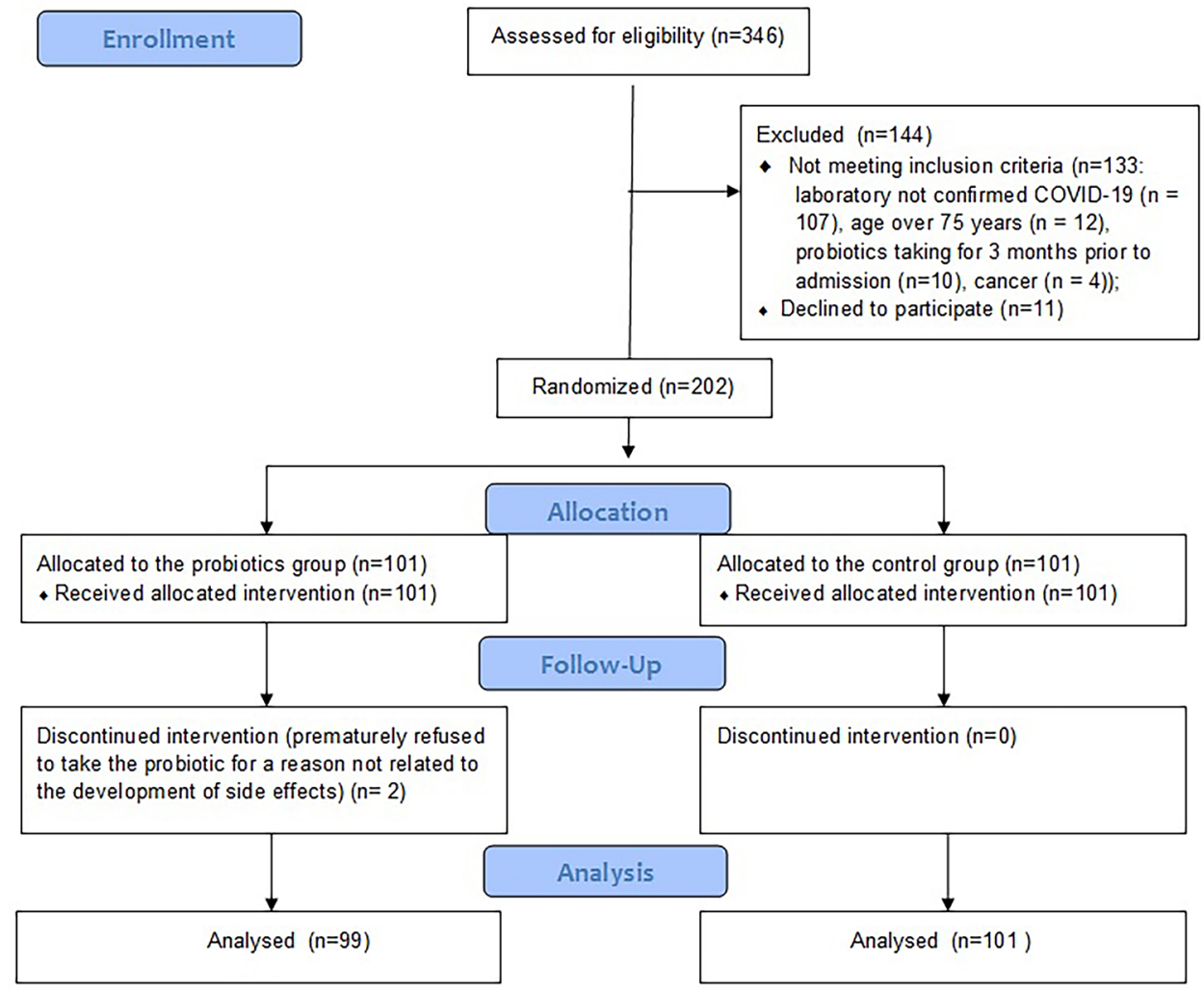


Table 1 Main characteristics and outcomes of patients who received probiotics (PRO group) and who did not (CON group)

\begin{tabular}{llll}
\hline & Group PRO $(n=99)$ & Group CON $(n=101)$ & $p$ \\
\hline Age, years & $65(59-71)$ & $64(54-70)$ & 0.283 \\
Male/female & $44 / 55$ & $48 / 53$ & 0.662 \\
Body temperature at admission, ${ }^{\circ} \mathrm{C}$ & $37.3(36.9-37.7)$ & $37.2(36.8-37.6)$ & 0.657 \\
${\text { Body mass index, } \mathrm{kg} / \mathrm{m}^{2}}^{2}$ & $30.5(27.4-35.3)$ & $31.2(27.1-33.5)$ & 0.910 \\
Time from symptom onset to inclusion, days & $8(6-12)$ & $9(7-11)$ & 0.504 \\
Length of hospital stay, days & $11(10-14)$ & $11(9-14)$ & 0.440 \\
Total duration of disease, days & $20(18-24)$ & $21(18-25)$ & 0.471 \\
Death, $n(\%)$ & $4(4.0 \%)$ & $4(4.0 \%)$ & 0.491 \\
Admission to intensive care unit, $n(\%)$ & $5(5.1 \%)$ & $7(6.9 \%)$ & 0.576 \\
The need for mechanical ventilation, $n(\%)$ & $4(4.0 \%)$ & $5(5.0 \%)$ & 0.976 \\
Oxygen support, $n$ (\%) & $47(47.5 \%)$ & $44(43.6 \%)$ & 0.579 \\
Duration of oxygen support, days & $6(2-11)$ & $7(1-11)$ & 0.513 \\
Patients with diarrhea on admission, $n(\%)$ & $12(12.1 \%)$ & $11(10.9 \%)$ & 0.785 \\
Duration of diarrhea on admission, days & $2(1-4)$ & $4(3-6)$ & 0.049 \\
Patients with hospital-acquired diarrhea, $n(\%)$ & $4(4.0 \%)$ & $10(9.9 \%)$ & 0.109 \\
Duration of hospital-acquired diarrhea, days & $4(4-6)$ & $5(3-6)$ & 0.777 \\
\hline
\end{tabular}

on the course of COVID-19 in the aforementioned patients, except for a tendency towards a decrease in mortality rate in patients with cardiovascular diseases (Table S2; Fig. 2b, c).

Probiotic administration did not exert significant effects on the course of COVID-19 in the subgroups of patients who had $<25 \%, 25-50 \%$, and $>50 \%$ lung involvement (Table S3), except for a decrease in frequency of admission to the intensive care unit in the group of patients with $25-50 \%$ lung involvement $(0.0 \%$ vs. $10.9 \%$; $p=0.024)$.

The administration of the probiotic did not exert a significant effect on the course of COVID-19 in subgroups of patients delineated based on age younger or older than 65 years (Table $\mathrm{S} 4$ ). In addition, no significant differences were observed between patients with serum CRP levels less

Table 2 Change in the values of the main biomarkers between the beginning (point 1) and end (point 2) of the trial in patients who received probiotics (PRO group) and those who did not (CON group)

\begin{tabular}{|c|c|c|c|c|c|c|c|c|}
\hline \multirow{2}{*}{$\frac{\text { Group }}{\text { Biomarker }}$} & \multicolumn{3}{|c|}{ PRO group $(n=99)$} & \multicolumn{3}{|c|}{ CON group $(n=101)$} & \multirow{2}{*}{$\frac{p^{* *}}{1}$} & \multirow{2}{*}{$\frac{p^{* *}}{2}$} \\
\hline & 1 & 2 & $p^{*}$ & 1 & 2 & $p^{*}$ & & \\
\hline Lung lesion volume, $\%$ & $50[50-75]$ & $50[50-75]$ & 0.453 & $50[50-75]$ & $50[50-75]$ & 0.547 & 0.393 & 0.570 \\
\hline $\mathrm{C}$-reactive protein, $\mathrm{mg} / \mathrm{L}$ & $66[24-116]$ & $3[1-5]$ & $<0.001$ & $58[28-108]$ & $3[1-5]$ & $<0.001$ & 0.784 & 0.680 \\
\hline White blood cells, $10^{9} / \mathrm{L}$ & $5.5[4.0-7.7]$ & $8.2[6.7-10.0]$ & $<0.001$ & $6.1[4.6-9.7]$ & $8.6[6.3-11.9]$ & $<0.001$ & 0.035 & 0.210 \\
\hline Neutrophils, $10^{9} / \mathrm{L}$ & $4.1[2.6-5.9]$ & $6.2[4.2-7.4]$ & 0.002 & $4.6[2.9-8.5]$ & $6.3[4.5-9.5]$ & 0.019 & 0.053 & 0.231 \\
\hline Lymphocytes, $10^{9} / \mathrm{L}$ & $1.0[0.7-1.4]$ & 1.4 [1.0-1.9] & $<0.001$ & $1.0[0.7-1.3]$ & $1.2[0.9-1.8]$ & $<0.001$ & 0.740 & 0.656 \\
\hline Platelets, $10^{9} / \mathrm{L}$ & $226[171-272]$ & $311[250-392]$ & $<0.001$ & $236[168-316]$ & $316[227-398]$ & 0.001 & 0.697 & 0.615 \\
\hline $\mathrm{ESR}, \mathrm{mm} / \mathrm{L}$ & $25[21-28]$ & $20[14-25]$ & 0.001 & 24 [20-27] & $20[14-24]$ & 0.001 & 0.446 & 0.903 \\
\hline Creatinine, $\mu \mathrm{mol} / \mathrm{L}$ & $96[80-110]$ & 83 [76-94] & $<0.001$ & 96 [84-109] & 83 [75-100] & $<0.001$ & 0.632 & 0.752 \\
\hline ALT, U/L & $30[20-42]$ & $40[32-64]$ & $<0.001$ & $31[22-46]$ & 37 [22-82] & $<0.001$ & 0.367 & 0.516 \\
\hline AST, U/L & 35 [29-49] & $36[25-51]$ & 0.626 & $35[28-48]$ & $43[22-53]$ & 0.404 & 0.548 & 0.530 \\
\hline Albumin, g/L & $41[39-44]$ & $37[31-43]$ & 0.450 & $41[40-44]$ & $35[30-37]$ & 0.221 & 0.230 & 0.270 \\
\hline Total bilirubin, $\mu \mathrm{mol} / \mathrm{L}$ & $9[6-11]$ & $8[6-10]$ & 0.605 & $10[7-12]$ & $11[9-14]$ & 0.579 & 0.386 & 0.051 \\
\hline LDH, U/L & $541[453-687]$ & $453[392-564]$ & $<0.001$ & 533 [410-698] & $441[384-568]$ & $<0.001$ & 0.578 & 0.538 \\
\hline Ferritin, $\mu \mathrm{g} / \mathrm{L}$ & 442 [224-639] & 469 [347-793] & 0.617 & 436 [208-749] & 501 [211-779] & 0.067 & 0.923 & 0.593 \\
\hline Fibrinogen, $\mathrm{g} / \mathrm{L}$ & $6.0[5.1-7.4]$ & $3.5[2.8-3.9]$ & $<0.001$ & $5.8[4.7-7.4]$ & $3.5[2.9-4.5]$ & $<0.001$ & 0.283 & 0.376 \\
\hline Potassium, mmol/L & $4.5[4.1-4.9]$ & $5.1[4.5-5.5]$ & 0.037 & $4.4[4.1-4.7]$ & $4.9[4.6-5.4]$ & 0.034 & 0.433 & 0.849 \\
\hline
\end{tabular}

ESR erythrocyte sedimentation rate, $A L T$ alanine aminotransferase, $A S T$ aspartate aminotransferase, $L D H$ lactate dehydrogenase

*Difference between the beginning (point 1) and end (point 2) of the trial within the groups

** Difference between groups at the beginning (point 1) and end (point 2) of the trial 
Table 3 Maximum or minimum values of the main biomarkers during the trial

\begin{tabular}{lccc}
\hline & PRO group $(n=99)$ & CON group $(n=101)$ & $p$ \\
\hline $\begin{array}{l}\text { The maximum value during the trial } \\
\text { Lung lesion volume, } \%\end{array}$ & $50[50-75]$ & & \\
C-reactive protein, mg/L & $83[53-125]$ & $50[50-75]$ & 0.531 \\
White blood cells, $10^{9} / \mathrm{L}$ & $8.9[7.5-12.0]$ & $74[45-128]$ & 0.398 \\
Neutrophils, $10^{9} / \mathrm{L}$ & $7.0[5.3-10.0]$ & $10.0[7.3-14.2]$ & 0.268 \\
ESR, mm/L & $28[24-32]$ & $7.6[5.0-11.6]$ & 0.500 \\
Creatinine, $\mu \mathrm{mol} / \mathrm{L}$ & $96[83-110]$ & $27[22-30]$ & 0.132 \\
ALT, U/L & $38[23-85]$ & $99[84-112]$ & 0.509 \\
AST, U/L & $43[30-59]$ & $43[33-74]$ & 0.964 \\
Total bilirubin, mmol/l & $10[7-12]$ & $44[29-58]$ & 0.396 \\
LDH, U/L & $652[542-793]$ & $11[9-14]$ & 0.313 \\
Ferritin, $\mu \mathrm{g} / \mathrm{L}$ & $489[321-762]$ & $633[464-830]$ & 0.394 \\
Fibrinogen, g/L & $6.3[5.4-7.6]$ & $518[212-988]$ & 0.753 \\
The minimum value during the trial & $6.3[4.9-7.7]$ & 0.504 \\
Albumin, g/L & $37[31-42]$ & & \\
Lymphocytes, 10 $9 / \mathrm{L}$ & $0.7[0.6-1.1]$ & $34[30-37]$ & 0.057 \\
\hline
\end{tabular}

ESR erythrocyte sedimentation rate, $A L T$ alanine aminotransferase, $A S T$ aspartate aminotransferase, $L D H$ lactate dehydrogenase or greater than $60 \mathrm{mg} / \mathrm{L}$ on admission (Table S5) (this is the cut-off value for severe systemic inflammation according to the local guidelines [26]). However, a more pronounced decrease in lymphocyte count was observed in the PRO group among patients with CRP level less than $60 \mathrm{mg} / \mathrm{L}$.

There were no cases of infections caused by these probiotic strains.

\section{Diarrhea in COVID-19}

Diarrhea on admission was observed in 23 (11.5\%) patients; no significant difference was observed between the PRO and CON groups in this regard (Table 1). It was observed that diarrhea ceased earlier in patients who received the probiotic than in those who did not (Table 1).

Hospital-acquired diarrhea developed in 14 (7.0\%) patients, and only in those who received antibiotics (14/154
$(9.1 \%)$ vs. $0 / 46(0.0 \%) ; p=0.034)$. Probiotic intake in the general cohort tended to prevent the development of hospital-acquired diarrhea. However, a significant difference was observed in the group that received only one antibiotic [ $n=71$; hospital-acquired diarrhea incidence: 0 vs. $4(12.5 \%) ; p=0.023$ ]. The probiotic was not found to be effective in preventing hospital-acquired diarrhea in patients who received more than one antibiotic simultaneously or sequentially ( $n=83$; hospital-acquired diarrhea incidence: $4(10.5 \%)$ vs. $6(13.3 \%) ; p=0.696)$.

Intake of the probiotic did not significantly affect the duration of hospital-acquired diarrhea (Table 1).

\section{Liver Injury in COVID-19}

Signs of liver injury were observed in 95 (47.5\%) patients on admission, with no significant difference between the PRO
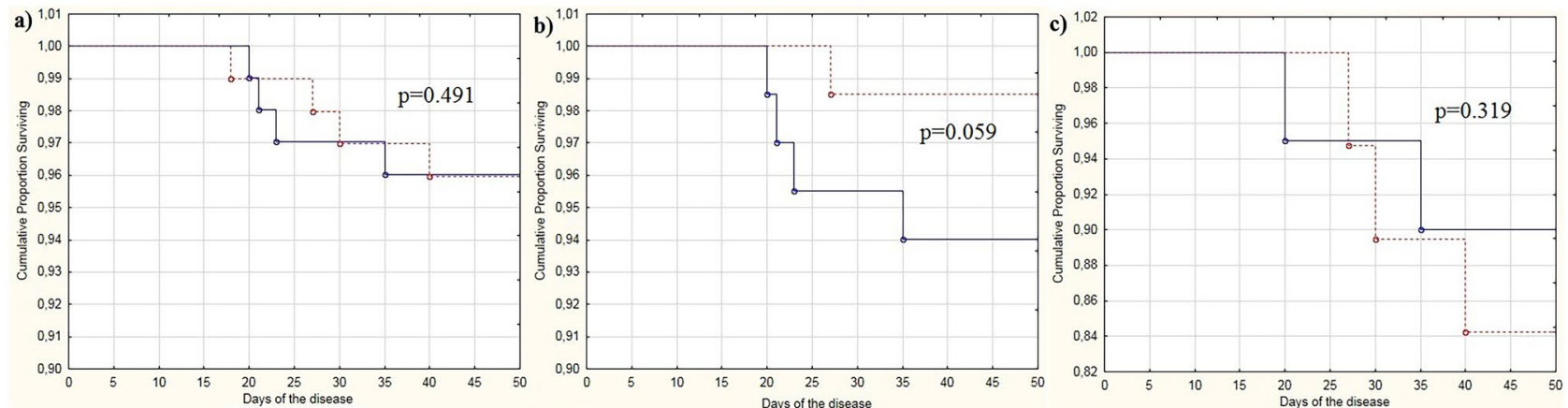

Fig. 2 Survival curves for patients with coronavirus disease (COVID-19) who received the probiotic (dotted line) and those who did not (control group) (solid line): (a) all patients, (b) patients with cardiovascular diseases, and (c) patients with diabetes mellitus 
Table 4 Patients with abnormal liver biomarker values

\begin{tabular}{|c|c|c|c|}
\hline & Group PRO $(n=99)$ & Group CON $(n=101)$ & $p$ \\
\hline \multicolumn{4}{|l|}{ Patients with abnormal liver biomarkers on admission } \\
\hline$\overline{\text { ALT }>\text { ULN (45 U/L) on admission, } n(\%)}$ & $21(21.2 \%)$ & $26(25.7 \%)$ & 0.450 \\
\hline ALT $>3 \mathrm{ULN}$ on admission, $n(\%)$ & $2(2.0 \%)$ & $1(1.0 \%)$ & 0.549 \\
\hline AST $>$ ULN (45U/L) on admission, $n(\%)$ & $27(27.3 \%)$ & $27(26.7 \%)$ & 0.932 \\
\hline AST $>3$ ULN on admission, $n(\%)$ & $3(3.0 \%)$ & $1(1.0 \%)$ & 0.303 \\
\hline Total bilirubin $>$ ULN $(21 \mu \mathrm{mol} / \mathrm{L})$ on admission, $n(\%)$ & $4(4.0 \%)$ & $4(4.0 \%)$ & 0.977 \\
\hline Serum albumin $<\mathrm{ULN}(35 \mathrm{~g} / \mathrm{L})$ on admission, $n(\%)$ & $10(10.1 \%)$ & $4(4.0 \%)$ & 0.889 \\
\hline ALP > ULN (360 U/L) on admission, $n(\%)$ & $3(3.0 \%)$ & $3(3.0 \%)$ & 0.980 \\
\hline GGT > ULN (60 U/L) on admission, $n(\%)$ & $11(11.1 \%)$ & $15(14.9 \%)$ & 0.432 \\
\hline Any abnormal liver biomarker, $n(\%)$ & $45(45.5 \%)$ & $50(49.5 \%)$ & 0.566 \\
\hline \multicolumn{4}{|l|}{ Patients with progression of pre-admission liver injury } \\
\hline $\begin{array}{l}\text { Increase in the ALT level during the trial in those with high ALT } \\
\text { levels at admission, } n(\%)\end{array}$ & $12(12.1 \%)$ & $12(11.9 \%)$ & 0.958 \\
\hline $\begin{array}{l}\text { Increase in the AST level during the trial in those with high AST } \\
\text { levels at admission, } n(\%)\end{array}$ & $4(4.0 \%)$ & $10(9.9 \%)$ & 0.105 \\
\hline Any progression of pre-admission liver injury, $n(\%)$ & $15(15.2 \%)$ & $16(15.8 \%)$ & 0.893 \\
\hline \multicolumn{4}{|l|}{ Patients who developed liver injury after admission } \\
\hline ALT $>$ ULN during the trial, $n(\%)$ & $25(25.3 \%)$ & $15(14.9 \%)$ & 0.066 \\
\hline ALT > 3ULN during the trial, $n(\%)$ & $5(5.1 \%)$ & $11(10.9 \%)$ & 0.128 \\
\hline AST > ULN during the trial, $n(\%)$ & $18(18.2 \%)$ & $9(8.9 \%)$ & 0.055 \\
\hline AST > 3ULN during the trial, $n(\%)$ & $1(1.0 \%)$ & $4(4.0 \%)$ & 0.182 \\
\hline Total bilirubin $>$ ULN during the trial, $n(\%)$ & $0(0.0 \%)$ & $2(2.0 \%)$ & 0.159 \\
\hline Serum albumin $<$ ULN during the trial, $n(\%)$ & $5(5.1 \%)$ & $6(5.9 \%)$ & 0.783 \\
\hline ALP $>$ ULN during the trial, $n(\%)$ & $0(0.0 \%)$ & $2(2.0 \%)$ & 0.159 \\
\hline GGT $>$ ULN during the trial, $n(\%)$ & $3(3.0 \%)$ & $5(5.0 \%)$ & 0.488 \\
\hline Any liver injury after admission, $n(\%)$ & $32(32.3 \%)$ & $23(22.8 \%)$ & 0.130 \\
\hline
\end{tabular}

$A L T$ alanine aminotransferase, $U L N$ upper limit of normal, $A S T$ aspartate aminotransferase, $A L P$ alkaline phosphatase, $G G T$ gamma-glutamyl transferase

and CON groups. Liver injury progressed during hospitalization in $31(15.5 \%)$ of these patients, and developed after admission in $55(27.5 \%)$ other patients; probiotic intake did not prevent liver injury in any of these cases (Table 4).

\section{Discussion}

The aim of our study was to evaluate the effect of a multistrain probiotic on mortality, disease course, respiratory function, diarrhea, and liver injury in COVID-19 patients in a randomized controlled trial. We could not confirm the findings of an Italian retrospective study which reported that probiotic supplementation could reduce mortality in a cohort of COVID-19 patients [23]. It should be noted that the probiotic composition we used was different from that used by the Italian research group, though it is unlikely that this difference in composition can explain the difference in study outcomes. Mortality was significantly higher in the above mentioned study than in the present study (22\% vs.
4\%). This significant difference in mortality between the two studies may be explained by the different strategies used for COVID-19 management. The Italian researchers mainly administered hydroxychloroquine, lopinavir, azithromycin, and tocilizumab in accordance with the clinical recommendations of that time (the study was performed between March and April 2020). In the present study, lopinavir was not used, azithromycin and hydroxychloroquine were used less frequently, and almost all patients were administered with dexamethasone and anticoagulants.

The second factor responsible for the difference in mortality may be the differences in ethnicity, resulting in differences in the interaction between the virus and the host, as well as the differences in the strain composition in different countries. A third factor may be selection bias that is characteristic of retrospective studies. In addition, the severity of disease could have differed among patients in the two studies.

The differences in mortality between the patients who received and did not receive probiotics almost reached the limits of significance in the subgroup of patients with 
chronic cardiovascular diseases $(1.5 \%$ vs. $6.0 \% ; p=0.059)$. Notably, there were no patients with chronic cardiovascular diseases in the above mentioned study by Ceccarelli et al. [23]. It is interesting that gut microbiota has been shown to play a role in the development of heart failure [27]. A study with a larger sample size should be conducted to examine the effect of probiotics on mortality in this subgroup of patients and to test the hypothesis that probiotics may reduce mortality in patients with chronic cardiovascular diseases.

The probiotic did not have a significant effect on the course of the disease, inflammatory biomarkers, and renal dysfunction in our study. Moreover, this finding was observed not only in the general cohort of patients, but also in the following subgroups: subgroups of patients with chronic cardiovascular diseases, diabetes, and different volumes of lung damage; subgroups delineated based on age younger or older than 65 years; and subgroups defined based on the presence or absence of severe systemic inflammation on admission.

In our study, the administration of probiotics shortened the duration of COVID-19-associated diarrhea. This result is in agreement with the results of a retrospective study by d'Ettorre et al. [24]. It should be noted that the combination of probiotics used in the two studies differed. The causes of diarrhea in COVID-19 patients were heterogeneous [6]; viral diarrhea which develops in the early days of the disease as well as antibiotic-associated diarrhea which develops later and often in the hospital (hospital-acquired diarrhea) were observed. We also studied the effect of the multi-strain probiotic on the incidence and duration of hospital-acquired diarrhea. Hospital-acquired diarrhea developed only in those patients who received antibiotics, which may confirm its antibiotic-associated nature. Although hospital-acquired diarrhea was less common among patients who received the probiotic than among those who did not, this difference was not significant in the general cohort of patients. However, it was significant in the subgroup of patients who received only one antibiotic. Additionally, the administration of the probiotic did not affect the duration of hospital-acquired diarrhea.

Thus, in our study, probiotics for COVID-19 showed small but distinctly positive effects; these effects included a shortening of the duration of viral diarrhea by an average of 2 days, and the prevention of hospital-acquired diarrhea in patients who received a single antibiotic.

The bacteria included in the probiotic therapy used in this study have been reported to be useful in the treatment of antibiotic-associated diarrhea [28] and acute viral diarrhea $[29,30]$ in previous trials. These effects may be associated with their ability to form biofilms on the surface of the gut epithelium, which prevents colonization by Clostridioides difficile [31]. This in turn inhibits the growth and toxinogenesis of these and other pathogenic bacteria [32]. Intestinal viral infections cause increased permeability of the intestinal barrier [33]; probiotic bacteria have been reported to reduce the permeability of the intestinal barrier, and normalize the functioning of ion transporters in the epithelial cell membrane [34]. Thus, our results in patients with COVID-19 are consistent with the findings from the studies cited above.

The main strength of our study is that this is the first randomized controlled study investigating the effect of probiotics on a wide range of indicators in COVID-19. In addition, we analyzed these effects not only in the general cohort of patients, but also in subgroups within the cohort. We also performed a detailed analysis of the effects of probiotic supplementation on liver injury associated with COVID19. However, there are several limitations to our study. This was a single-center open-label study, and did not include a placebo group. We used a probiotic that was different from those used in earlier studies, which may also be interpreted as a limitation. In addition, it was not possible to differentiate between the adverse effects of the probiotic and COVID19-related signs due to the pronounced polymorphism of the manifestations of this disease. However, there were no cases of infections caused by these probiotic strains in our study.

New randomized controlled trials that include populations from different countries are needed to confirm our findings.

\section{Conclusion}

In conclusion, the studied probiotic did not have a significant effect on mortality and changes in most biomarkers in COVID-19 patients. However, the probiotic showed potential as treatment for diarrhea associated with COVID-19, and for the prevention of diarrhea in patients who receive a single antibiotic as part of COVID-19 treatment. A larger study on the effect of probiotics in COVID-19 patients with chronic cardiovascular diseases should be conducted to test the hypothesis that probiotics may reduce mortality in this cohort of patients.

Supplementary Information The online version contains supplementary material available at https://doi.org/10.1007/s12602-021-09858-5.

Acknowledgements We would like to express our gratitude to the doctors, nurses, and other staff of the Tareev Clinic of Internal Diseases who took part in this study.

Author Contribution The idea and design of the study were developed by Vladimir Ivashkin and Elena Poluektova. Material preparation, data collection, and analysis were performed by all authors. The first draft of the manuscript was written by Roman Maslennikov, and all authors commented on previous versions of the manuscript. All authors read and approved the final manuscript. 
Availability of Data and Material The datasets analyzed during the current study may be available from the corresponding author on reasonable request.

\section{Declarations}

Ethics Approval The study was approved by the local ethics committee (Conclusion №. 34-20 of September 9, 2020) in accordance with the Declaration of Helsinki.

Consent to Participate Informed consent was obtained from all individual participants included in the study.

Consent for Publication Patients' personal data are not published.

Conflicts of Interest The authors declare no competing interests.

\section{References}

1. Mohamadian M, Chiti H, Shoghli A et al (2021) COVID-19: virology, biology and novel laboratory diagnosis. J Gene Med 23:e3303. https://doi.org/10.1002/jgm.3303

2. Umakanthan S, Sahu P, Ranade AV et al (2020) Origin, transmission, diagnosis and management of coronavirus disease 2019 (COVID-19). Postgrad Med 96:753-758. https://doi.org/10.1136/ postgradmedj-2020-138234

3. Trush EA, Poluektova EA, Beniashvilli AG et al (2020) The evolution of human probiotics: challenges and prospects. Probiotics Antimicrob Proteins 12:1291-1299. https://doi.org/10.1007/s12602-019-09628-4

4. Zolnikova O, Komkova I, Potskherashvili N et al (2018) Application of probiotics for acute respiratory tract infections. Ital J Med 12:32-38. https://doi.org/10.4081/itjm.2018.931

5. Tariq R, Saha S, Furqan F et al (2020) Prevalence and mortality of COVID-19 patients with gastrointestinal symptoms: a systematic review and meta-analysis. Mayo Clin Proc 95:1632-1648

6. Maslennikov R, Poluektova E, Ivashkin V et al (2021) Diarrhoea in adults with coronavirus disease-beyond incidence and mortality: a systematic review and meta-analysis. Infect Dis (Lond) 53:348-360. https://doi.org/10.1080/23744235.2021.1885733

7. Maslennikov R, Ivashkin V, Ufimtseva A, Poluektova E (2021) A clinical variant of coronavirus disease 2019 with diarrhoea as the initial symptom compared with other variants. Minerva Gastroenterol (Torino). https://doi.org/10.23736/S2724-5985.21.02827-0

8. Megyeri K, Dernovics Á, Al-Luhaibi ZII, Rosztóczy A (2021) COVID-19-associated diarrhea. World J Gastroenterol 27:32083222. https://doi.org/10.3748/wjg.v27.i23.3208

9. Agamennone V, Krul CAM, Rijkers G et al (2018) A practical guide for probiotics applied to the case of antibiotic-associated diarrhoea in the Netherlands. BMC Gastroenterol 18:103. https:// doi.org/10.1186/s12876-018-0831-x

10. Cangemi DJ, Lacy BE (2019) Management of irritable bowel syndrome with diarrhoea: a review of nonpharmacological and pharmacological interventions. Ther Adv Gastroenterol 12:1-19. https://doi.org/10.1177/1756284819878950

11. Isolauri E (2003) Probiotics for infectious diarrhoea Gut 52:436-437. https://doi.org/10.1136/gut.52.3.436

12. Wijarnpreecha $K$, Ungprasert $P$, Panjawatanan $P$ et al (2021) COVID19 and liver injury: a meta-analysis. Eur J Gastroenterol Hepatol 33:990-995. https://doi.org/10.1097/MEG.0000000000001817

13. Maslennikov R, Ivashkin V, Efremova I, Poluektova E, Shirokova E (2021) Probiotics in hepatology: an update. World J Hepatol 13:1154-1166 https://doi.org/10.4254/wjh.v13.i9.1154
14. Mirzaei R, Attar A, Papizadeh S et al (2021) The emerging role of probiotics as a mitigation strategy against coronavirus disease 2019 (COVID-19). Arch Virol 166:1819-1840. https://doi.org/ 10.1007/s00705-021-05036-8

15. Singh K, Rao A (2021) Probiotics: a potential immunomodulator in COVID-19 infection management. Nutr Res 87:1-12. https://doi.org/10.1016/j.nutres.2020.12.014

16. Patra S, Saxena S, Sahu N et al (2021) Systematic network and meta-analysis on the antiviral mechanisms of probiotics: a preventive and treatment strategy to mitigate SARS-CoV-2 infection. Probiotics Antimicrob Proteins 13:1138-1156. https://doi. org/10.1007/s12602-021-09748-w

17. Mullish BH, Marchesi JR, McDonald JAK et al (2021) Probiotics reduce self-reported symptoms of upper respiratory tract infection in overweight and obese adults: should we be considering probiotics during viral pandemics? Gut Microbes 13:1-9. https://doi.org/10.1080/19490976.2021.1900997

18. Manna S, Chowdhury T, Chakraborty R, Mandal SM (2021) Probiotics-derived peptides and their immunomodulatory molecules can play a preventive role against viral diseases including COVID-19. Probiotics Antimicrob Proteins 13:611-623. https:// doi.org/10.1007/s12602-020-09727-7

19. Olaimat AN, Aolymat I, Al-Holy M et al (2020) The potential application of probiotics and prebiotics for the prevention and treatment of COVID-19. NPJ Sci Food 4:17. https://doi.org/10. 1038/s41538-020-00078-9

20. Mahooti M, Miri SM, Abdolalipour E, Ghaemi A (2020) The immunomodulatory effects of probiotics on respiratory viral infections: a hint for COVID-19 treatment? Microb Pathog 148:104452. https://doi.org/10.1016/j.micpath.2020.104452

21. Bozkurt HS, Quigley EM (2020) The probiotic Bifidobacterium in the management of Coronavirus: a theoretical basis. Int $\mathrm{J}$ Immunopathol Pharmacol 34:2058738420961304. https://doi. org/10.1177/2058738420961304

22. Li Q, Cheng F, Xu Q et al (2021) The role of probiotics in coronavirus disease-19 infection in Wuhan: a retrospective study of 311 severe patients. Int Immunopharmacol 95:107531. https:// doi.org/10.1016/j.intimp.2021

23. Ceccarelli G, Borrazzo C, Pinacchio C et al (2021) Oral bacteriotherapy in patients with COVID-19: a retrospective cohort study. Front Nutr 7:613928. https://doi.org/10.3389/fnut.2020.613928

24. d'Ettorre G, Ceccarelli G, Marazzato M et al (2020) Challenges in the management of SARS-CoV2 infection: the role of oral bacteriotherapy as complementary therapeutic strategy to avoid the progression of COVID-19. Front Med (Lausanne) 7:389. https:// doi.org/10.3389/fmed.2020.00389

25. Li B, Yang J, Zhao F et al (2020) Prevalence and impact of cardiovascular metabolic diseases on COVID-19 in China. Clin Res Cardiol 109:531-538. https://doi.org/10.1007/s00392-020-01626-9

26. Kamkin E et al. (2020) Interim guidelines for the prevention, diagnosis and treatment of new coronaviral infection (COVID19). https://static-0.minzdrav.gov.ru/system/attachments/attaches/ 000/051/777/original/030902020_COVID-19_v8.pdf. Accessed 03 Sept 2020

27. Ivashkin V, Fadeeva M, Skhirtladze M et al (2020) Intestinal microbiota in the pathogenesis of chronic heart failure. Ital J Med 14:1-8. https://doi.org/10.4081/itjm.2020.1185

28. Szajewska H, Kołodziej M (2015) Systematic review with metaanalysis: Lactobacillus rhamnosus GG in the prevention of antibiotic-associated diarrhoea in children and adults. Aliment Pharmacol Ther 42:1149-1157. https://doi.org/10.1111/apt.13404

29. Li YT, Xu H, Ye JZ (2019) Efficacy of Lactobacillus rhamnosus GG in treatment of acute pediatric diarrhea: a systematic review with meta-analysis. World J Gastroenterol 25:4999-5016. https:// doi.org/10.3748/wjg.v25.i33.4999 
30. Di JB, Gai ZT (2020) Protective efficacy of probiotics on the treatment of acute rotavirus diarrhea in children: an updated metaanalysis. Eur Rev Med Pharmacol Sci 24:9675-9683. https://doi. org/10.26355/eurrev_202009_23057

31. Rasinkangas P, Tytgat HLP, Ritari J et al (2020) Characterization of highly mucus-adherent non-GMO derivatives of Lacticaseibacillus rhamnosus GG. Front Bioeng Biotechnol 8:1024. https:// doi.org/10.3389/fbioe.2020.01024

32. Valdés-Varela L, Hernández-Barranco AM, Ruas-Madiedo P, Gueimonde M (2016) Effect of Bifidobacterium upon Clostridium difficile growth and toxicity when co-cultured in different prebiotic substrates. Front Microbiol 7:738. https://doi.org/10.3389/ fmicb.2016.00738
33. Kawahara T, Makizaki Y, Oikawa Y et al (2017) Oral administration of Bifidobacterium bifidum G9-1 alleviates rotavirus gastroenteritis through regulation of intestinal homeostasis by inducing mucosal protective factors. PLoS One 12:e0173979. https://doi. org/10.1371/journal.pone.0173979

34. Kumar A, Hecht C, Priyamvada S (2014) Probiotic Bifidobacterium species stimulate human SLC26A3 gene function and expression in intestinal epithelial cells. Am J Physiol Cell Physiol 307:C1084-C1092. https://doi.org/10.1152/ajpcell.00194.2014

Publisher's Note Springer Nature remains neutral with regard to jurisdictional claims in published maps and institutional affiliations. 